\title{
Plasmon-enhanced fluorometry based on gold nanostructure arrays. Method and device
}

\author{
V.I. Chegel, V.K. Lytvyn, A.M. Lopatynskyi, P.E. Shepeliavyi, O.S. Lytvyn, Yu.V. Goltvyanskyi \\ V. Lashkaryov Institute of Semiconductor Physics, NAS of Ukraine, \\ 41, prospect Nauky, 03028 Kyiv, Ukraine; \\ Phone: +38(044)525-56-26, e-mail:vche111@yahoo.com,lop2000@ukr.net,lytvet@ukr.net
}

\begin{abstract}
In this work, we describe a method of surface-enhanced fluorometry, based on the phenomenon of localized surface plasmon resonance in unordered gold nanostructure arrays. The theoretical approach for the model system "gold nanoparticle-dielectric spacer" in the electrostatic approximation by solution of Laplace's equation is considered. The developed technology for manufacturing the plasmonic substrates as well as design of the novel laser-based compact fluorometer are presented. The arrays of gold nanostructures on solid substrates (nanochips) coated with different thicknesses of $\mathrm{SiO}_{2}$ were developed and fabricated by thermal annealing of gold island films with subsequent dielectric spacer deposition. As an example for verification of the proposed method, the fluorescence properties of the system "gold nanostructures array - $\mathrm{SiO}_{2}$ dielectric coating - Rhodamine $6 \mathrm{G}$ " were studied. It has been shown that enhancement of dye emission up to 22 times for dielectric coating with the thickness of about $20 \mathrm{~nm}$ is possible. Presented method is of importance for the development of the novel nanoscale sensors, biomolecular assays and nanoplasmonic devices.
\end{abstract}

Keywords: localized surface plasmon resonance, plasmonic nanostructures, plasmonenhanced fluorescence, rhodamine $6 \mathrm{G}$.

Manuscript received 03.03.15; revised version received 16.06.15; accepted for publication 03.09.15; published online 30.09.15.

\section{Introduction}

Today, the number of works related to the investigation of fluorescence, one of the most subtle and complicated optical phenomena, is increasing rapidly. This is caused by the prospects for using fluorescence as a basis of rapid methods for detection and identification of chemical and biological substances. Fluorescence is one of the most widely used methods in biochemistry [1], clinical testing [2] and cellular imaging [3]. The capabilities of fluorescence technology are now exploited for quantitative and qualitative analysis and for biological research in general [4]. For example, structure of cells and their organelles, ion fluxes in cells and membrane processes have been investigated with application of fluorescent probes [5]. Additionally, fluorescence methods are being increasingly used for studies of conformational changes of biomolecules and specific interactions between them [6]. Fluorescence technique can also be an effective tool in development of new medical treatments [7] and drug delivery methods [8]. A large number of studies using fluorescence methods in biochemistry and molecular biology have been focused on using dyes to visualize individual components of biological systems $[9,10]$.

Many researches reported on the phenomenon of plasmon-enhanced fluorescence (PEF), which is induced by localized surface plasmon resonance (LSPR) that appears in high-conductive metal (plasmonic) nanostructures (PN) [11] and can significantly increase the emission of fluorophore $[12,13]$. In particular, a large change in light emission of fluorescent molecule 
located near PN is caused by high electromagnetic field around gold and silver PN under LSPR conditions [14]. A number of active structures have been developed for PEF, primarily based on silver colloids $[15,16]$, silver and gold island films $[17,18]$, and ordered noble metal nanoparticle arrays [19].

In order to observe PEF, fluorescent molecule should be located near to the LSPR-generating surface, i.e. in the PN near-field zone [20]. However, very close disposition of fluorophore and PN leads to fluorescence quenching caused by the energy transfer from the excited molecule to the metallic surface [21]. Common approach reported in literature to overcome this issue is to hold the fluorophore away from a nanostructured surface at an optimal distance by a dielectric coating, which provides stable PEF conditions [22]. Another approach depends on preparation of the complex: PN chemical linker - biomolecule - dye, which provides the required distance from dye to nanoparticle [23]. These techniques make it possible to obtain up to 50 -fold fluorescence enhancement [24].

In this paper, we describe the method for plasmonenhanced fluorometry and technology for producing gold nanochips aimed at in-practice realization of the enhancement mechanism. Presented here are the experimental results of studying the enhanced fluorescence signal of organic dye Rhodamine 6G (R6G) located at different distances from gold nanostructures, which was realized by using $\mathrm{SiO}_{2}$ dielectric coatings. We also report on the application of developed and patented by authors [25] compact laser-based fluorometer "Fluorotest ${ }^{\text {Nano" }}$ to detect the enhanced fluorescence emission of the dye using the above-mentioned method. We believe that presented methodology will contribute to the ongoing search for the combination of fluorescence spectroscopy methods, nanoplasmonics and nanotechnology in order to establish the possibilities of plasmon-enhanced fluorescence towards building highly efficient PEF-sensors.

\section{Theory and modeling}

The relationship between the plasmon-induced electromagnetic near-field of a metal nanostructure and properties of the adjacent dye has been previously studied in [26]. The fluorophore emission was demonstrated to change dramatically when it is placed on the different distances within PN, depending on the electric field. There are multiple factors that affect the molecule fluorescence signal: type of material, size and shape of the nanostructure, type of material and thickness of the nanoparticle coating, incident and emitted light wavelengths and intrinsic quantum yield of the fluorophore [27]. At the present time, scientists agree that there are two main factors affecting the fluorescence changes by plasmonic nanostructures. First of them is the plasmon field generated around PN by the incident light that, depending on wavelength, can enhance the excitation of the fluorophore, which, in turn, determines the level of fluorescence emission. The second one is the $\mathrm{PN}$-fluorophore interaction that reduces the ratio of radiative to non-radiative decay rate and, depending on the presence of the dielectric layer, influences the quantum yield of the fluorophore, resulting in fluorescence quenching [28].

Several mathematical models have been developed to describe the plasmon electric field around PN [20, 29], including an approach considering dielectriccoated PN [27], which is of special importance for PEF modeling. It is based on the electrostatic approximation by solution of Laplace's equation with the boundary conditions appropriate to the selected model [29] and takes into account only the dipole plasmon mode excited in spherical PN (Fig. 1).

The model shown in Fig. 1 uses the spherical coordinate system. According to this simplified approach [27], where the system has an azimuthal symmetry $\left(\frac{d E_{p}}{d \phi}\right)=0$, plasmon electric field $\left(E_{p}\right)$ at an observation point $r$, which is generated by the incident light $\left(E_{0}\right)$ in the vicinity of PN (with a radius $r_{1}$ ) coated with a dielectric shell (with thickness $r_{2}-r_{1}$ ), can be described by the following equation

$$
\begin{aligned}
& \vec{E}_{p}=\left[2 \frac{\varepsilon_{2} \varepsilon_{a}-\varepsilon_{3} \varepsilon_{b}}{\varepsilon_{2} \varepsilon_{a}+2 \varepsilon_{3} \varepsilon_{b}} \cdot\left(\frac{r_{2}}{r}\right)^{3}+1\right] \cdot \vec{E}_{0} \cdot \cos (\theta) \cdot \vec{e}_{r}+ \\
& +\left[\frac{\varepsilon_{2} \varepsilon_{a}-\varepsilon_{3} \varepsilon_{b}}{\varepsilon_{2} \varepsilon_{a}+2 \varepsilon_{3} \varepsilon_{b}} \cdot\left(\frac{r_{2}}{r}\right)^{3}+1\right] \cdot \vec{E}_{0} \cdot \sin (\theta) \cdot \vec{e}_{\theta},
\end{aligned}
$$

and the electric field inside the shell $\left(\vec{E}_{p}^{\text {layer }}\right)$ is

$$
\begin{aligned}
& \vec{E}_{p}^{\text {layer }}=\frac{3 \varepsilon_{3}\left[\left(\varepsilon_{1}+2 \varepsilon_{2}\right)+2\left(\varepsilon_{1}-\varepsilon_{2}\right) \cdot\left(\frac{r_{2}}{r}\right)^{3}\right]}{\varepsilon_{2} \varepsilon_{a}+2 \varepsilon_{3} \varepsilon_{b}} \times \\
& \times \vec{E}_{0} \cdot \cos (\theta) \cdot \vec{e}_{r}-\frac{3 \varepsilon_{3}\left[\left(\varepsilon_{1}+2 \varepsilon_{2}\right)-2\left(\varepsilon_{1}-\varepsilon_{2}\right) \cdot\left(\frac{r_{2}}{r}\right)^{3}\right]}{\varepsilon_{2} \varepsilon_{a}+2 \varepsilon_{3} \varepsilon_{b}} \times \\
& \times \vec{E}_{0} \cdot \sin (\theta) \cdot \vec{e}_{\theta},
\end{aligned}
$$

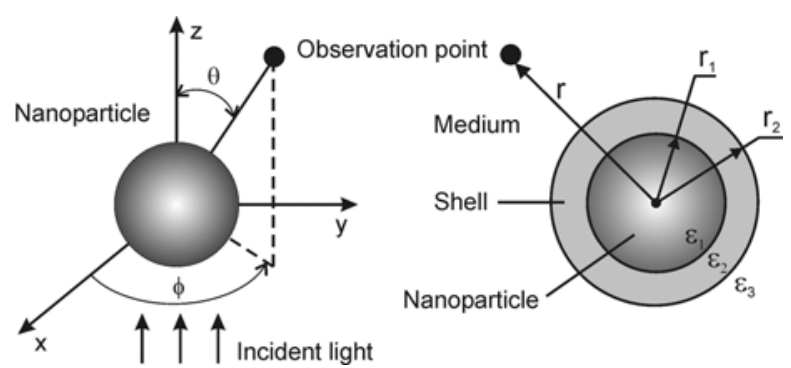

Fig. 1. Model of a dielectric-coated spherical PN placed in surrounding medium with a coordinate system used for the presentation of plasmon electric field. 
where $\vec{e}_{r}, \vec{e}_{\theta}$ are the unit vectors in $r$ and $\theta$ directions of the spherical coordinates, respectively,

$\varepsilon_{a}=\varepsilon_{1}(3-2 P)+2 \varepsilon_{2} P, \varepsilon_{b}=\varepsilon_{1} P+\varepsilon_{2}(3-P)$,

$P=1-\left(\frac{r_{1}}{r_{2}}\right)^{3}$,

$\varepsilon_{1}, \varepsilon_{2}, \varepsilon_{3}$ and $\varepsilon_{0}$ are the dielectric permittivity values of $\mathrm{PN}$, shell, outer surrounding medium and vacuum, respectively. For $\mathrm{PN}, \varepsilon_{1}$ is wavelength- and sizedependent and can be described by the Drude-Lorentz model [30]:

$\varepsilon_{1}(\omega)=\varepsilon_{0}\left(1-\omega_{p}^{2} \frac{1}{\omega^{2}+i \omega \gamma_{f}}+\omega_{p}^{2} \frac{1}{\omega_{0}^{2}-\omega^{2}+i \omega \gamma_{b}}\right)$,

where $\omega$ is the angular frequency of the incident light, $\omega_{0}-$ bound electron resonant frequency, $\omega_{p}-$ plasma frequency,

$\gamma_{f}=1 / \tau_{f}=1 / \tau_{0}+v_{f} / r_{1}, \gamma_{b}=1 / \tau_{b}$,

where $\tau_{f}$ and $\tau_{b}$ are the free electron relaxation time and bound electron decay time, respectively, $v_{f}$ is the Fermi velocity and $\tau_{0}$ - free electron scattering time for the bulk material.

Quantum yield $q$ indirectly influenced by the plasmon field $E_{p}$ [27] can be described as

$\frac{q}{q_{0}}=\frac{\frac{\gamma_{r}}{\gamma_{r}^{0}}}{\frac{\gamma_{r}}{\gamma_{r}^{0}}+\frac{\gamma_{a b s}}{\gamma_{r}^{0}}+\frac{1-q^{0}}{q_{0}}}$,

where $\gamma_{r}$ and $\gamma_{r}^{0}$ are the radiative decay rates in the presence and absence of PN, respectively, $\gamma_{a b s}$ is the non-radiative decay rate resulting from the energy absorbed by PN [31], and $q^{0}-$ intrinsic quantum yield of the fluorophore.

Therefore, fluorescence emission enhancement $(\Phi)$ is the combined effect of the excitation rate enhancement and quantum yield change, both influenced by the plasmon field, and it can be described as

$\Phi=\left(\frac{E_{p}}{E_{0}}\right)^{2} \frac{q}{q^{0}}$.

The electric field intensity near the 100-nmdiameter spherical gold nanoparticle with a 20 -nm-thick $\mathrm{SiO}_{2}$ shell has been calculated using the abovementioned theoretical approach implemented in the MathCAD software. The results of simulation are presented in Fig. 2 as the spectral distribution of average electric field intensity enhancement in the 3-nm vicinity around the spherical gold nanoparticle with a coating, where fluorophore molecules can reside. This system exhibits the enhancement of incident electric field intensity up to approximately 18 times with a maximum enhancement at the wavelength of $542 \mathrm{~nm}$.

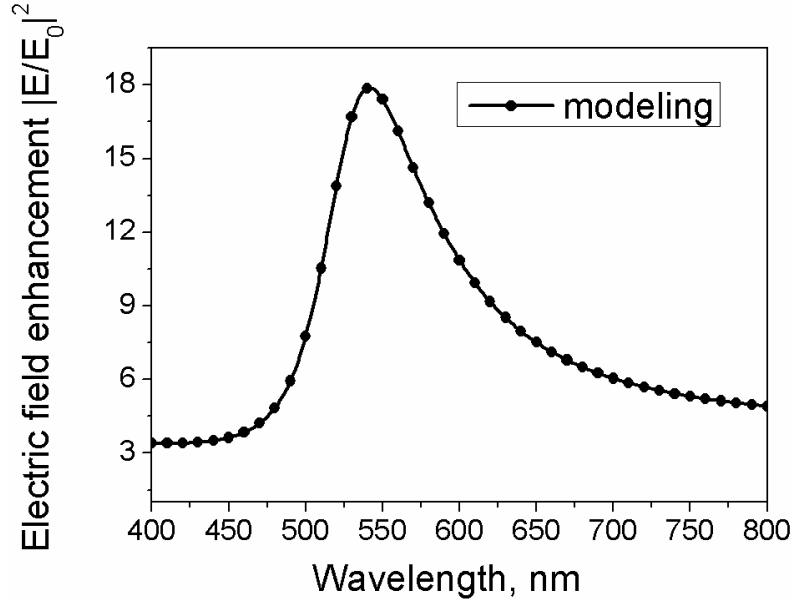

Fig. 2. Simulated spectral distribution of average electric field intensity enhancement in the 3-nm vicinity around the $100-\mathrm{nm}$ diameter spherical gold nanoparticle with a 20 -nm-thick $\mathrm{SiO}_{2}$ shell.

\section{Sample preparation}

Microscope glass slides $(13 \times 25 \mathrm{~mm})$ were used as substrates. Before metal film deposition, substrates were cleaned in the ultrasonic bath with surfactant, then treated in "piranha solution" for $30 \mathrm{~min}$ (caution this mixture is potentially explosive and it will became very hot), triply rinsed with a copious amount of deionized water and finally dried using $\mathrm{N}_{2}$ flow.

Gold island film fabrication and $\mathrm{SiO}_{2}$ coating were carried out by thermal vacuum evaporation method using the UVN-2M setup (pressure $10^{-3} \mathrm{~Pa}$ ) with deposition speed of about 0.11 to $0.14 \mathrm{~nm} / \mathrm{s}$. The mass thickness of gold island film of about $10 \mathrm{~nm}$ was chosen as the value that allows to obtain the separated welldefined nanostructures after annealing $\left(450^{\circ} \mathrm{C}, 2\right.$ hours in air atmosphere). To prepare the dielectric coating, $\mathrm{SiO}_{2}$ layers with a thickness of 10 to $25 \mathrm{~nm}$ in $5-\mathrm{nm}$ steps were deposited. Resulting structures (i.e. PN with the dielectric coating) were covered with fluorescent organic dye R6G by dipping them for $30 \mathrm{~s}$ into R6G aqueous solution with the dye concentration of $10^{-5} \mathrm{~mol} / \mathrm{L}$ and subsequent drying under room conditions. The presence of $\mathrm{R} 6 \mathrm{G}$ layer on glass or $\mathrm{SiO}_{2}$ surface was provided by opposite charges of $\mathrm{SiO}_{2}$ and $\mathrm{R} 6 \mathrm{G}$ in aqueous solution $[32,34]$.

\section{Experimental methods}

Absorbance spectra of gold island films, PN without coating and $\mathrm{PN}$ with $\mathrm{SiO}_{2}$ coating were measured using LSPR spectrometer "NanoPLASMON-003" [34]. Atomic force microscopy (AFM) measurements of nanochip samples were carried out on atomic force microscope NanoScope IIIa Dimension 3000. Developed by the authors portable laser-based fluorometer 
"Fluorotest ${ }^{\text {Nano" }}$ was used for steady-state fluorescence measurements (see schematic measurement setup and common view of the device in Fig. 3). The device has three built-in laser light sources $(405,532,650 \mathrm{~nm})$ and four LEDs $(360,470,535,632 \mathrm{~nm})$ that allows working both with nanochips and solutions in photometric cuvettes.

Fluorescence measurements of R6G layers on PN were carried out with the 532-nm green laser used as an excitation light source. The measurement scheme for fluorescence spectroscopy based on right-angle geometry with the 540-nm cut-off interference filter is depicted in Fig. 4. The simplified scheme of gold PN with the $\mathrm{SiO}_{2}$ dielectric coating, which provides fluorescence signal enhancement for the adjacent dye molecule, is shown in Fig. 4. The distance between the fluorophore molecules and $\mathrm{PN}$ was determined by the thickness of the dielectric $\mathrm{SiO}_{2}$ coating deposited on PN.

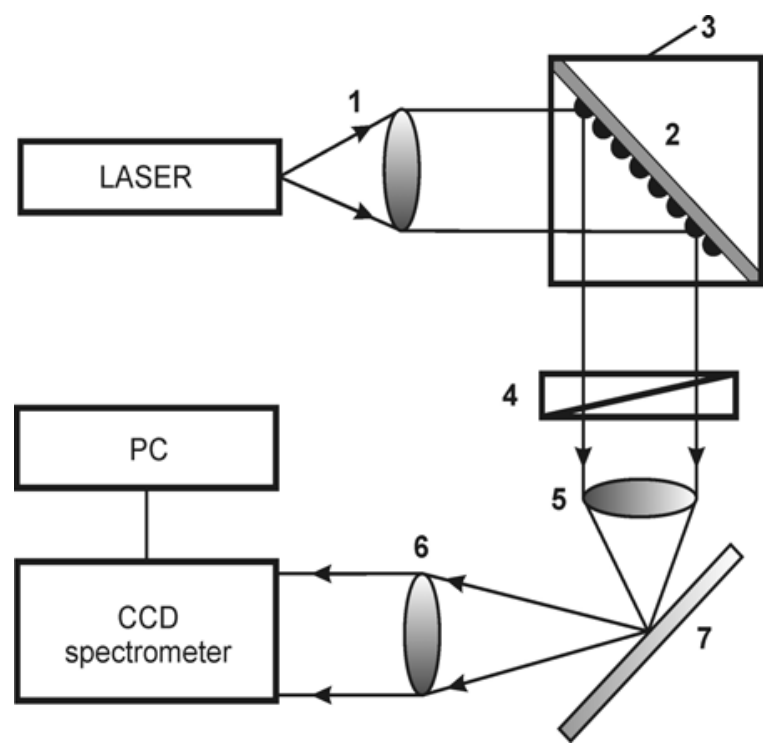

a)

\section{Results and discussion}

The top view AFM image of the gold island film produced according to the protocol described in Section 3 is shown in Fig. 5a. AFM measurements demonstrate that the maximal mass thickness of island film is about $12 \mathrm{~nm}$, which is close to the value specified in the fabrication protocol. Gold PN (Fig. 5b) after the thermal annealing of gold island film exhibit the shape of semi-ellipsoids with a height and an equivalent radius distributed within the ranges of $30 \ldots 80 \mathrm{~nm}$ and $20 \ldots 100 \mathrm{~nm}$, respectively. The average height of $\mathrm{PN}$ is about $60 \mathrm{~nm}$ and the average equivalent radius is close to $50 \mathrm{~nm}$.

Typical absorbance spectra of bare gold PN and gold $\mathrm{PN}$ with $\mathrm{SiO}_{2}$ coating of a different thickness $(10 \ldots 25 \mathrm{~nm})$ measured in air are shown in Fig. 6. All spectra reveal expressed plasmonic peak at $550 \mathrm{~nm}$ (for bare PN) and 580..610 nm (for covered PN). The observed LSPR position red shift accompanying the dielectric coating thickness increase confirms the plasmonic nature of registered absorbance peak [35].

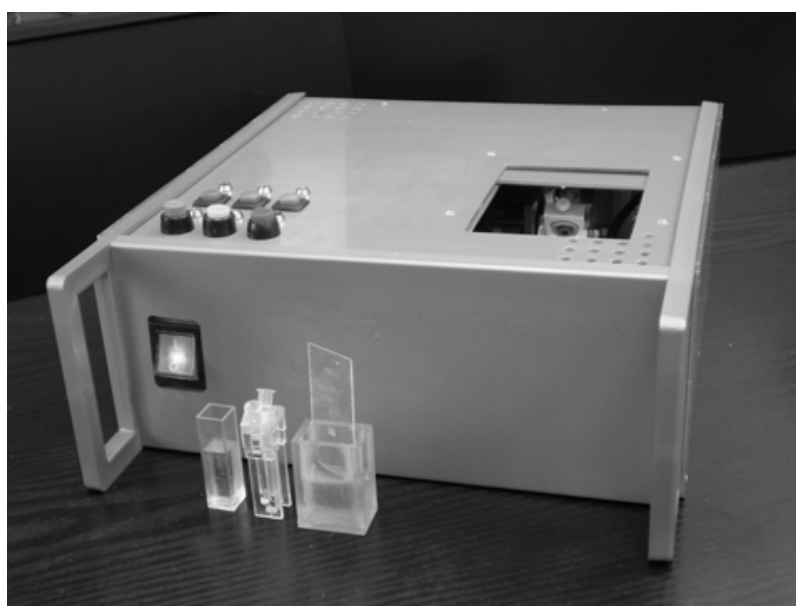

b)

Fig. 3. (a) Schematic of experimental setup for plasmon-enhanced fluorescence measurements. 1, 5, 6- lenses, 2- nanochip, 3 - sample holder, 4 - longpass filter, 7 - mirror. (b) Photo of the "Fluorotest ${ }^{\text {Nano" }}$ device.

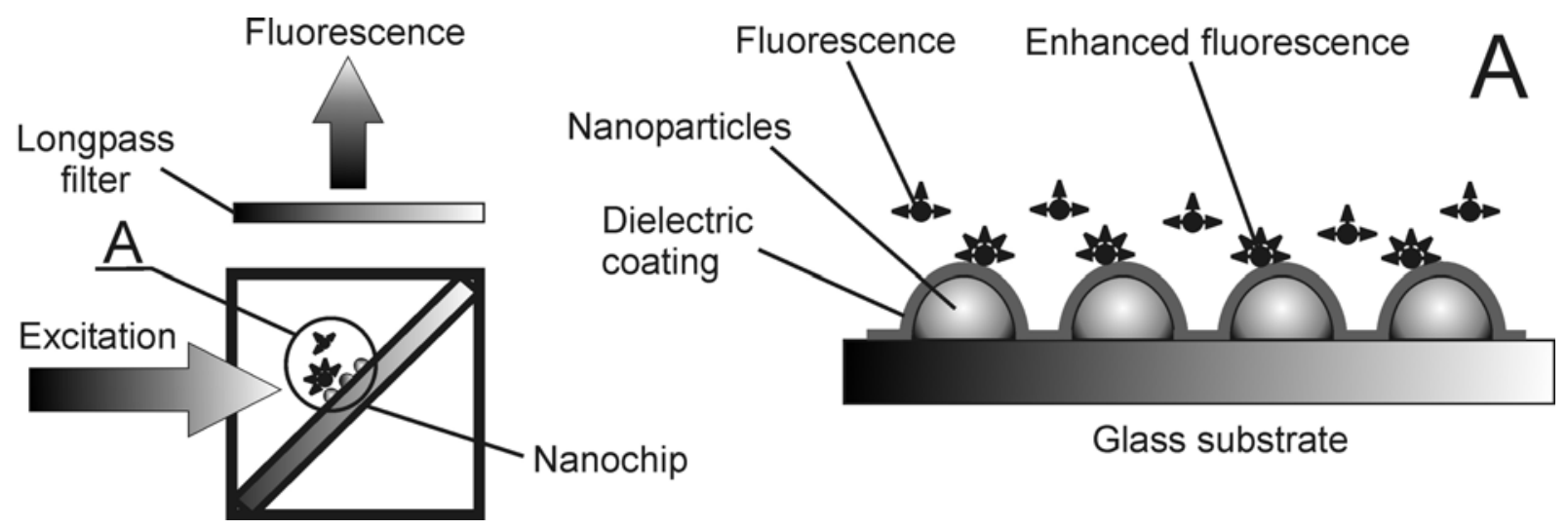

Fig. 4. Measurement scheme for $\mathrm{R} 6 \mathrm{G}$ plasmon-enhanced fluorescence detection near gold $\mathrm{PN}$ with $\mathrm{SiO}_{2}$ dielectric coating. 


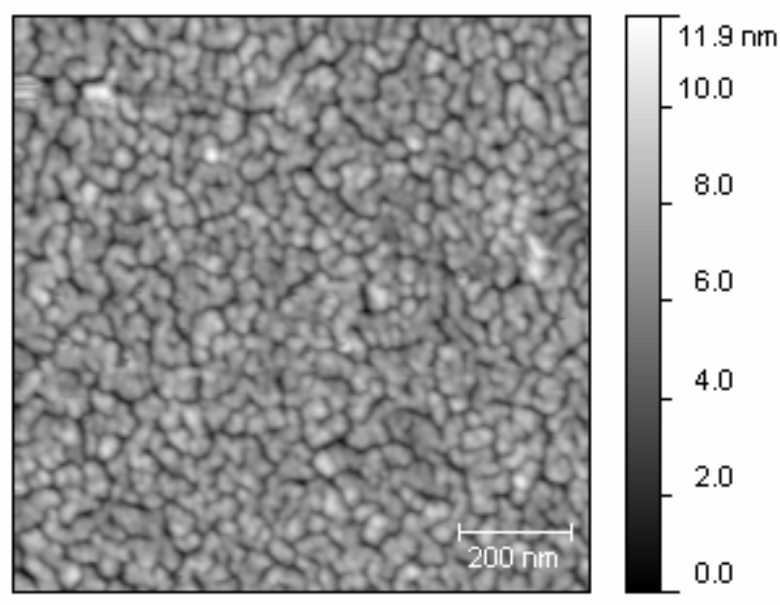

a)

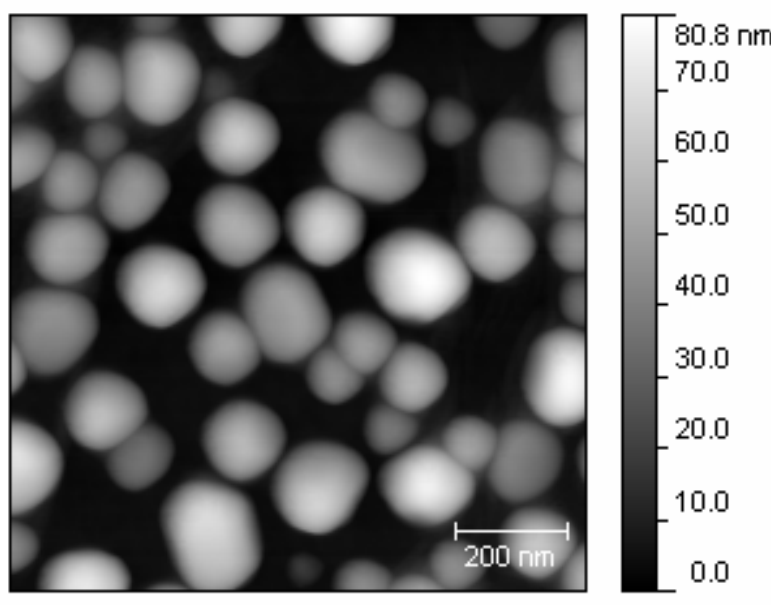

b)

Fig. 5. Top view AFM images of (a) gold island film and (b) gold PN.

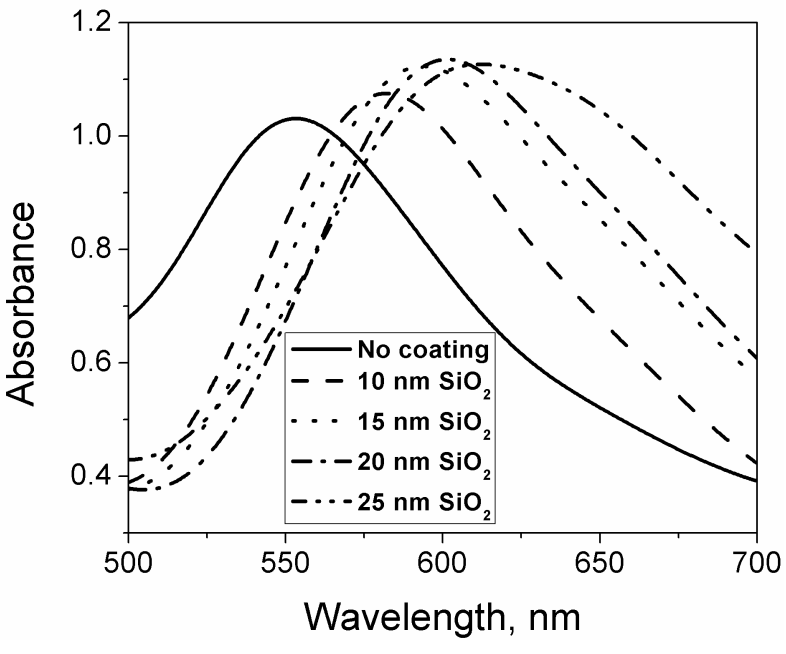

Fig. 6. Absorbance spectra of bare gold PN and gold PN with $\mathrm{SiO}_{2}$ dielectric coating having the thickness within the range of $10 \ldots .25 \mathrm{~nm}$.

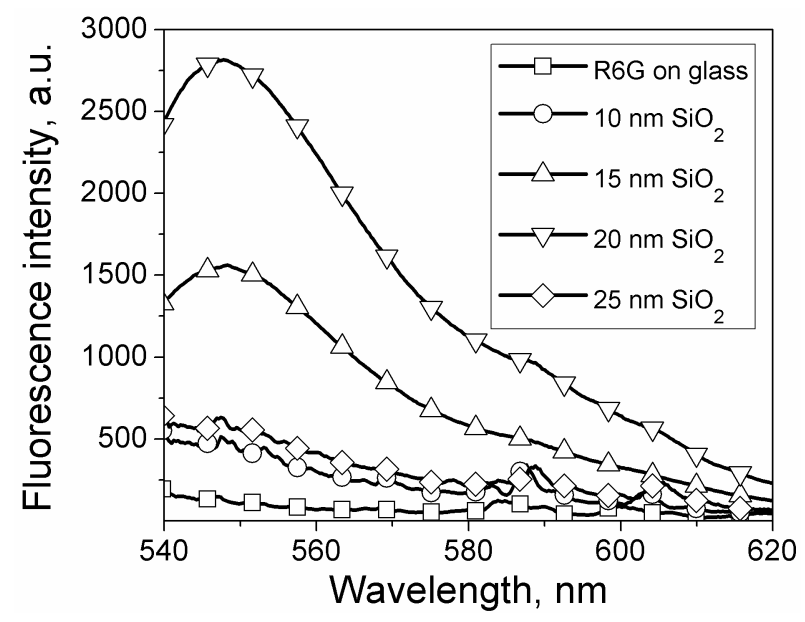

a)
All the studied PN samples covered with $\mathrm{SiO}_{2} /$ dye exhibit R6G fluorescence enhancement in comparison with the signal obtained from similar samples without PN (Fig. 7a). At the same time, the relationship between the intensity of fluorescent signal and $\mathrm{SiO}_{2}$ thickness is evidently nonlinear with a distinct peak. Namely, maximum R6G fluorescence was obtained for the sample with a 20 -nm-thick $\mathrm{SiO}_{2}$ coating. Fig. $7 \mathrm{~b}$ shows the fluorescence enhancement factor dependence on the thickness of dielectric coating, which exhibits the bellshaped character. Here, enhancement factor is estimated as a ratio of R6G fluorescence intensities for gold PN with $\mathrm{SiO}_{2}$ shell and bare glass substrate near maximum emission wavelength $(548 \mathrm{~nm})$. Small blue-shift of R6G emission with respect to tabular value can be explained by presence of silicon oxide shell near the dye, which changes the dielectric environment of R6G [36].

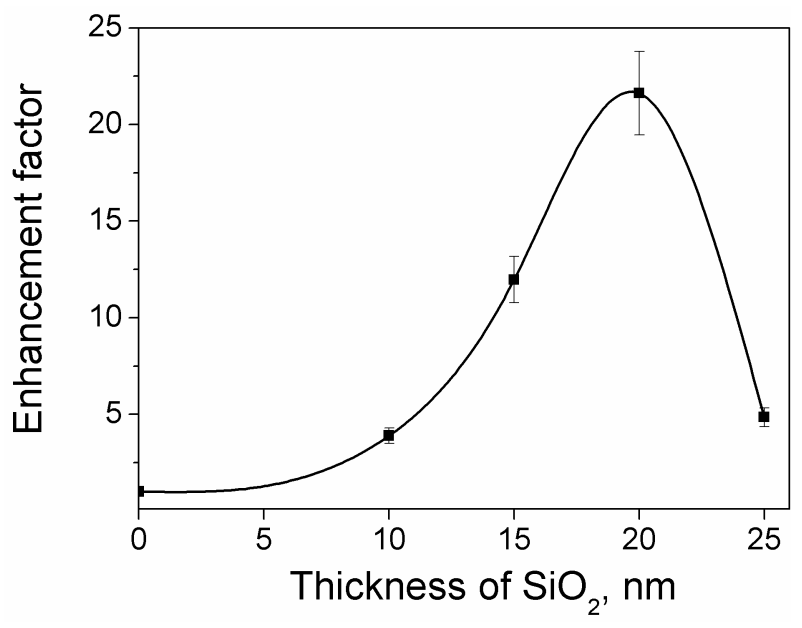

b)

Fig. 7. (a) Fluorescence spectra of R6G dye placed on the surface of gold $\mathrm{PN}$ coated with $\mathrm{SiO}_{2}$ layer of various thicknesses. (b) $\mathrm{R} 6 \mathrm{G}$ fluorescence enhancement factor dependence on the $\mathrm{SiO}_{2}$ thickness. 
It is of importance that the enhanced signal was observed only if the proximity of wavelengths of PN LSPR, dye emission and dye excitation has been provided, which is in agreement with well-known phenomenon of emission enhancement by strong electric field generated by PN under LSPR conditions [37]. The observed increase of dye emission using the proposed nanochips opens the possibility to provide plasmonenhanced fluorescence for flow mode real-time measurements with low analyte consumption. The experimental results of R6G fluorescence enhancement close to 22 times, obtained on the fabricated nanochips with $20-\mathrm{nm} \mathrm{SiO}_{2}$ coating, are in agreement with the results of modeling. It should be noted that the measured and calculated absorbance spectra of PN have some difference in the wavelength positions. This disagreement can be attributed to the presence of the dielectric substrate and the different sizes and shape of PN on the real nanochip. In particular, nanoparticles on the nanochip have rather semi-ellipsoid, not a spherical shape. Disagreement in enhancement values obtained in the model and the experimental data can also be caused by a simplified model "nanoparticle - dielectric coating dye". In the applied model, the quantum yield of the dye that affects the value of the energy transfer between the LSPR nanoparticle and dye molecules has not been considered, as well as interaction between nanostructures in array, this will be the part of our future work.

\section{Conclusions}

The method of fluorescence signal enhancement based on exploitation of plasmon-supporting nanochips was developed, which provides up to 22-fold emission enhancement for Rhodamine 6G. The relevant experimental studies have been carried out on the novel laser-based fluorometer "Fluorotest ${ }^{\text {Nano" }}$ that provides plasmonic control of fluorescence signal in the visible spectral region. Nanochips for plasmon-enhanced fluorescence based on gold nanostructures with dielectric coating of different thickness were designed and fabricated by thermal annealing of gold island films with subsequent $\mathrm{SiO}_{2}$ layer deposition. As a result of R6G fluorescence intensity measurements for different thicknesses of dielectric spacer, the optimal distance between dye molecules and plasmonic nanostructures providing a dominance of fluorescence enhancement over quenching was found. The obtained results demonstrate that the creation of "gold nanostructure dielectric spacer - fluorophore" system with precisely tailored optical and geometrical parameters and distinct resonant properties enables the development of highsensitive optoelectronic nanosensors for the analysis of weak fluorescence signals, e.g. for biochemical and diagnostic applications.

\section{Acknowledgements}

This work was supported by the Science and Technology Center in Ukraine (project 6044 for 2015 to 2017).

\section{References}

1. K. Aslan, I. Gryczynski, J. Malicka, Metal-enhanced fluorescence: an emerging tool in biotechnology // Current Opinion in Biotechnology, 16(1), p. 55-62 (2005).

2. O. Stranik, H.M. McEvoy, C. McDonagh, B.D. MacCraith, Plasmonic enhancement of fluorescence for sensor applications // Sensors and Actuators B, 107, p. 148-153 (2005).

3. M. Fernández-Suárez and A.Y. Ting, Fluorescent probes for super-resolution imaging in living cells // Molecular Cell Biology, 9(12), p. 929-943 (2008).

4. J.R. Lakowicz, Radiative decay engineering: Biophysical and biomedical applications // Anal. Biochem. 298(1), p. 1-24 (2001).

5. A.P. Demchenko, Introduction to Fluorescence Sensing. Springer, Netherlands, 2009.

6. S. Weiss, Fluorescence spectroscopy of single biomolecules // Science, 283(5408), p. 1676-1683 (1999).

7. A. Sieroń, K. Sieroń-Stoltny, A. Kawczyk-Krupka, W. Latos, S. Kwiatek, D. Straszak, A.M. Bugaj, The role of fluorescence diagnosis in clinical practice // Dove Press Journal: Onco Targets and Therapy, 2013(6), p. 977-982 (2013).

8. A. Agarwal, P.K. Tripathi, S. Tripathi, N.K. Jain, Fluorescence imaging: applications in drug delivery research // Current Drug Targets, 9(10), p. 895-899 (2008).

9. J. Rao, A. Dragulescu-Andrasi and H. Yao, Fluorescence imaging in vivo: recent advances // Current Opinion in Biotechnology, 18, p. 17-25 (2007).

10. G.P. Drummen, Fluorescent probes and fluorescence (microscopy) techniques - illuminating biological and biomedical research // Molecules, 17(12), p. 14067-14090 (2012).

11. N.L. Dmitruk, A.V. Goncharenko, E.F. Venger, Optics of Small Particles and Composite Media. Naukova dumka, Kyiv, 2009.

12. F. Tam, G.P. Goodrich, B.R. Johnson, Plasmonic enhancement of molecular fluorescence // Nano Lett. 7(2), p. 496-501 (2007).

13. M. Iosin, P. Baldeck, S. Astilean, Plasmonenhanced fluorescence of dye molecules // Nucl. Instrum. and Meth. in Phys. Res. 267, p. 403-405 (2009).

14. K. Drozdowicz-Tomsia, Gold and silver nanowires for fluorescence enhancement, Chap. 14 in: Nanowires - Fundamental Research Book, Ed. A. Hashim, p. 309-332, InTech, 2011.

15. S. Kruszewski, T. Wybranowski, M. Cyrankiewicz, Enhancement of FITC fluorescence by silver colloids and silver island films // Acta Physica Polonica, 6(113), p. 1599-1608 (2008).

16. C.D. Geddes, A. Parfenov, D. Roll, Electrochemical and laser deposition of silver for use in metalenhanced fluorescence // Langmuir, 15(5), p. 62366241 (2003). 
17. S. Xu, Y. Cao, J. Zhou, Plasmonic enhancement of fluorescence on silver nanoparticle films // Nanotechnology, 22, 275715 (2011).

18. R. Bardhan, N.K. Grady, J.R. Cole, Fluorescence enhancement by $\mathrm{Au}$ nanostructures: nanoshells and nanorods // ACS Nano, 3(3), p. 744-752 (2009).

19. V.I. Chegel, Nanostructured materials for biosensor applications: comparative review of preparation methods, Chap. 13 in: Manipulation of Nanoscale Materials: An Introduction to Nanoarchitectonics, Ed. K. Ariga, p. 318-355, RSC Nanoscience \& Nanotechnology, vol. 24, Cambridge, UK, 2012.

20. A. Pascal, P. Bharadwaj and L. Novotny, Enhancement and quenching of single-molecule fluorescence // Phys. Rev. Lett. 96(11), 113002 (2006).

21. E. Fort and S. Grésillon, Surface enhanced fluorescence // J. Phys. D: Appl. Phys. 41(1), 013001 (2008).

22. O.G. Tovmachenko, C. Graf, D.J. van den Heuvel, Fluorescence enhancement by metal-core/silicashell nanoparticles // Adv. Mater. J. 18, p. 91-95 (2006).

23. J. Zhang, Y. Fu, M.H. Chowdhury and J.R. Lakowicz, Metal-enhanced single-molecule fluorescence on silver particle monomer and dimer: coupling effect between metal particles // Nano Lett. 7(7), p. 2101-2107 (2007).

24. P. Bharadwaj, P. Anger and L. Novotny, Nanoplasmonic enhancement of single-molecule fluorescence // Nanotechnology, 18(4), 044017 (2007).

25. Patent Ukraine №75984, CI: G01N 21/00 (2012.01), G01N 21/64 (2006.01); 2012 / V.I. Chegel, O.P. Demchenko, V.I. Nazarenko, V.K. Lytvyn, A.M. Lopatynskyi, O.L. Kukla, O.S. Pavliuchenko, Optoelectronic biosensorfluorometer.

26. J.R. Lakowicz, M.H. Chowdhury, K. Ray et al., Plasmon-controlled fluorescence: a new detection technology // Proc. Soc. Photo Opt. Instrum. Eng. 6099, 609909 (2006).
27. K.A. Kang, J. Wang, J.B. Jasinski, Fluorescence manipulation by gold nanoparticles: from complete quenching to extensive enhancement // $J$. Nanobiotech. 9(16), p. 1-13 (2011).

28. T. Härtling, P. Reichenbach, L.M. Eng, Near-field coupling of a single fluorescent molecule and a spherical gold nanoparticle // Opt. Exp. 15(20), p. 12806-12817 (2007).

29. A.E. Neeves and M.H. Birnboim, Composite structures for the enhancement of nonlinear-optical susceptibility // J. Opt. Soc. Am. B, 6(4), p. 787-796 (1989).

30. M. Fox, Optical Properties of Solids. Oxford University Press, New York, 2001.

31. L. Novotny and B. Hecht, Principles of Nanooptics. Cambridge University Press, UK, 2012.

32. S.H. Behrens, D.G. Grier, The charge of glass and silica surfaces // J. Chem. Phys. 115, p. 6716-6721 (2001).

33. P.K. Paul, S.A. Hussain, D. Bhattacharjee and M. Pal, Adsorption of cationic laser dye onto polymer/surfactant complex film // Chin. J. Chem. Phys. 24(3), p. 348 (2011).

34. Patent Ukraine № 65947, CI: G01N 21/00 (2011.01), G01N 21/25 (2006.01), G01N 33/53 (2006.01); 2011 / V.I. Chegel, A.Y. Bieliaiev, J. Guo, A.M. Lopatinskyi, B. Lukas, O.L. Kukla, O.S. Pavliuchenko, Biosensor on basis of localized surface plasmon resonance.

35. H. Xu, M. Käll, Modeling the optical response of nanoparticle-based surface plasmon resonance sensors // Sensors and Actuators B, 87(2), p. 244249 (2002).

36. I. Trenkmann, S. Bok, V. Korampally, S. Gangopadhyay, H. Graaf, and C. von Borczyskowski, Counting single Rhodamine $6 \mathrm{G}$ dye molecules in organosilicate nanoparticles // Chem Phys. 406, p. 41-46 (2012).

37. Y. Chen, K. Munechika and D.S. Ginger, Dependence of fluorescence intensity on the spectral overlap between fluorophores and plasmon resonant single silver nanoparticles // Nano Lett. 7(3), p. 690696 (2007). 